\title{
Recombination and positive selection contribute to evolution of Listeria monocytogenes inlA
}

\section{Correspondence \\ M. Wiedmann \\ mw16@cornell.edu}

Received 21 February 2007

Revised 8 May 2007

Accepted 9 May 2007

\author{
R. H. Orsi, ${ }^{1}$ D. R. Ripoll, ${ }^{2}$ M. Yeung, ${ }^{3}+$ K. K. Nightingale ${ }^{1} \ddagger$
} and M. Wiedmann ${ }^{1}$

\author{
${ }^{1}$ Department of Food Science, Cornell University, Ithaca, NY, USA \\ ${ }^{2}$ Computational Biology Service Unit, Cornell Theory Center, Cornell University, Ithaca, NY, USA \\ ${ }^{3}$ Department of Microbiology and Immunology, Cornell University, Ithaca, NY, USA
}

\begin{abstract}
The surface molecule InIA interacts with E-cadherin to promote invasion of Listeria monocytogenes into selected host cells. DNA sequencing of inlA for $40 \mathrm{~L}$. monocytogenes isolates revealed 107 synonymous and 45 nonsynonymous substitutions. A frameshift mutation in a homopolymeric tract encoding part of the InIA signal peptide was identified in three lineage II isolates, which also showed reduced ability to invade human intestinal epithelial cells. Phylogenies showed clear separation of in/A sequences into lineages I and II. Thirteen in/A recombination events, predominantly involving lineage II strains as recipients (12 events), were detected and a number of amino acid residues were shown to be under positive selection. Four of the 45 nonsynonymous changes were found to be under positive selection with posterior probabilities $>95 \%$. Mapping of polymorphic and positively selected amino acid sites on the partial crystal structure for InIA showed that the internalin surface of the leucine-rich repeat (LRR) region that faces the InIA receptor E-cadherin does not include any polymorphic sites; all polymorphic and positively selected amino acids mapped to the outer face of the LRR region or to other InIA regions. The data show that (i) in/A is highly polymorphic and evolution of in/A involved a considerable number of recombination events in lineage II isolates; (ii) positive selection at specific amino acid sites appears to contribute to evolution of $i n / A$, including fixation of recombinant events; and (iii) single-nucleotide deletions in a lineage II-specific $3^{\prime}$ homopolymeric tract in inIA lead to complete loss of $\operatorname{In} \mid \mathrm{A}$ or to production of truncated $\operatorname{In} \mid \mathrm{A}$, which conveys reduced invasiveness. In conclusion, inlA has a complex evolutionary history, which is consistent with $L$. monocytogenes' natural history as an environmental pathogen with broad host-range, including its adaptation to environments and hosts where different in/A alleles may provide a selective advantage or where in/A may not be required.
\end{abstract}

\section{INTRODUCTION}

Listeria monocytogenes is a facultative intracellular foodborne pathogen, which can cause septicaemia, encephalitis,

tPresent address: Biological Sciences Department, Cal Poly State University, San Luis Obispo, CA 93407, USA

łPresent address: Department of Animal Sciences, 108B Animal Sciences Building, Colorado State University, Fort Collins, CO 8052311 , USA

Abbreviations: BEB, new Bayes empirical Bayes; IR, inter-repeat; LRRs, leucine-rich repeats; LRT, likelihood ratio test; MLST, multilocus sequence typing; NEB, naive empirical Bayes; PGS, proline/glycine-rich segment.

The GenBank/EMBL/DDBJ accession numbers for the in/A sequences of the isolates used in this study are EF445899-EF445938.

Supplementary tables showing the strains and primers used in this study as well as the details of recombination events in inlA allelic types are available with the online version of this paper. meningoencephalitis and abortion in different mammalian hosts, most commonly in humans and ruminants (Vazquez-Boland et al., 2001). L. monocytogenes can be divided into at least three distinct genetic lineages as determined by various molecular subtyping methods, including multilocus sequence typing (MLST) (Ward et al., 2004; Wiedmann et al., 1997; Nightingale et al., 2005a). Lineage I isolates have been responsible for the majority of listeriosis outbreaks and are more commonly isolated from human clinical cases than foods (Gray et al., 2004; Jeffers et al., 2001); lineage I thus has been hypothesized to represent a human-host-adapted lineage (Nightingale et al., 2005a). While lineage II strains have been isolated from sporadic human clinical cases, they are rarely responsible for human listeriosis outbreaks (Jeffers et al., 2001). Furthermore, lineage II strains are significantly more common among food isolates than among human clinical isolates (Gray et al., 2004), suggesting that 
these isolates may be better adapted to a non-host environment. Lineage III strains are predominantly isolated from animal clinical cases and are rarely isolated from environmental and food samples or human clinical cases (Jeffers et al., 2001; Roberts et al., 2006). L. monocytogenes lineages thus may have adapted to different host- and non-host-associated ecological niches.

Bacterial surface molecules involved in attachment to and invasion of host cells may play important roles in host specificity (e.g. Wilson et al., 2000). In L. monocytogenes, a group of cell surface proteins termed internalins, which are characterized by the presence of variable numbers of leucine-rich repeats (LRRs), appear to play important roles in attachment to host cells. While more than 25 internalin genes have been identified in L. monocytogenes, internalin A (InlA), encoded by inlA, has been shown to be particularly important for host-cell invasion (Lecuit et al., 2001). InlA is typically anchored to the bacterial cell wall via an LPXTG anchor (Dhar et al., 2000) and has been shown to bind to the host protein E-cadherin, which is an outer-membrane protein expressed in epithelial cells of several organisms, including humans (Mengaud et al., 1996). While InlA has been shown to be critical for invasion of intestinal epithelial cells and thus for crossing of the intestinal epithelial barrier, internalin A-E-cadherin interactions appear to be host-specific; InlA binds to human and guinea pig E-cadherin; however, a single amino acid difference in the murine E-cadherin (presence of glutamate in murine E-cadherin at aa 16 compared to a proline in humans and guinea pig E-cadherin) is sufficient to prevent InlA binding (Lecuit et al., 1999). In addition to its role in intestinal invasion, InlA may also contribute to crossing of the maternofetal barrier in pregnant hosts (Lecuit et al., 2004), even though these contributions were not apparent in a pregnant guinea pig model (Bakardjiev et al., 2004). InlA is 800 aa long and can be divided into eight distinct regions. Starting at the $\mathrm{N}$-terminal end, internalin $\mathrm{A}$ has a signal peptide followed by an $\alpha$-helix motif and fifteen and a half 22-residue LRRs, forming a right-handed solenoid with each repeat adding a helical turn (Schubert et al., 2002). C-terminal of the LRRs, the inter-repeat (IR) or immunoglobulin (Ig)-like region is the most flexible part of the internalin domain (Schubert et al., 2002). This region is followed by the $\mathrm{B}$ repeats, which consist of two repeats of 70 aa (B repeats 1 and 2) and a third repeat of 49 aa (B repeat 3) (Dramsi et al., 1993; Gaillard et al., 1991). The most C-terminal functionally important domain of InlA is the transmembrane region, which includes an LPXTG anchoring motif and a short positively charged tail. Additionally, a small region with no described function is located between the IR region and the B repeats and a proline/glycine-rich segment (PGS), also with no known function, is located between the $B$ repeats and the LPXTG motif (Dramsi et al., 1993; Gaillard et al., 1991).

In this work, we analysed the evolutionary history of inlA using $40 \mathrm{~L}$. monocytogenes isolates obtained from diverse sources, including human and bovine clinical cases, foods, and natural environments. An approach using a combination of evolutionary analyses and phenotypic characterization using tissue culture invasion assays showed that inlA has a complex evolutionary history involving positive selection, recombination among lineage II strains and single-nucleotide deletions that lead to reduced invasiveness in selected lineage II strains.

\section{METHODS}

Bacterial isolates and lysate preparation. A collection of $132 \mathrm{~L}$. monocytogenes isolates collected in New York State between 2000 and 2002, including 120 isolates from foods, human clinical cases and bovine clinical cases (previously described by Nightingale et al., 2005a) and 12 isolates from natural environments (Sauders et al., 2006) was stratified by source (human clinical cases, bovine clinical cases, food or natural environment) and ten isolates were randomly selected for each source. All isolates had previously been characterized by EcoRI ribotyping, and ribotyping data had also been used to classify isolates to lineage (Nightingale et al., 2005a; Sauders et al., 2006). The 40 isolates selected represented the three major $L$. monocytogenes genetic lineages: lineage I (18 isolates), lineage II (21 isolates) and lineage III (one isolate) (Supplementary Table S1, available with the online version of this paper, shows detailed information for all 40 isolates).

DNA sequencing. Four pairs of primers were used to amplify four fragments covering the whole inlA ORF (Supplementary Table S2, available with the online version of this paper, shows all primer sequences). PCR reactions were performed using $1 \times$ PCR buffer, $1.5 \mathrm{mM} \mathrm{MgCl}_{2}, 50 \mu \mathrm{M}$ dNTPs, $0.5 \mu \mathrm{M}$ of each primer and $0.05 \mathrm{U}$ Taq DNA polymerase $\mu \mathrm{l}^{-1}$ (Perkin Elmer-Applied Biosystems) as well as 1-2 $\mu$ l bacterial lysate. PCR products were purified using the QIAquick PCR purification kit (Qiagen) and DNA sequencing was performed at Macrogen using the same primers used for PCR. inlA sequences were assembled using Seqman (DNASTAR, Lasergene).

Descriptive sequence analysis. inlA contigs for the $40 \mathrm{~L}$. monocytogenes isolates were aligned with CLUSTAL W (MEGALIGN, DNASTAR, Lasergene) and all variable sites were proofread for errors. Descriptive analyses, including $\mathrm{G}+\mathrm{C}$ content, percentage of polymorphisms and number of synonymous and nonsynonymous substitutions, were carried out using DnaSP (Rozas \& Rozas, 1999).

Phylogenetic analysis. MODELTEST (Posada \& Crandall, 1998) was used to find the most likely model of DNA substitution. One sequence representing each of the $23 \mathrm{inlA}$ allelic types was used to construct rooted and unrooted maximum-likelihood phylogenetic trees in PAUP (http://paup.csit.fsu.edu/) using the nucleotide substitution model HKY85 $+\mathrm{G}+\mathrm{I}$, which was chosen by MODELTEST. Bootstrap analysis was performed with 100 repetitions using PAUP. The rooted tree that was constructed assuming a molecular clock and the unrooted tree that did not assume a molecular clock were used to test the molecular clock assumption using a likelihood ratio test (LRT) with $n-2$ degrees of freedom (with $n$ being the number of alleles).

Recombination analysis. The program GENECONV (http:// www.math.wustl.edu/ sawyer) was used to identify fragments that were likely to have originated by recombination. Recombination analyses were performed using an alignment that contained one sequence representing each inlA allelic type. The GENECONV analysis was repeated with four different 'gscale' values, including G0 (the default), G1, G2 and G3. Mismatch penalties increase from G1 to G3 and are highest for G0. GENECONV outputs were further analysed and 
fragments with the same breakpoints were considered to represent the same recombination event, as previously described (Nightingale et al., 2005a).

Positive selection analysis. The program codeml in the software package PAML version 3.14 (Yang, 1997) was used to test whether inlA is likely to include codons that are under positive selection and to identify specific amino acid sites that showed statistically significant evidence for positive selection (Yang et al., 2005). The naive empirical Bayes (NEB) inference approach was used for M3 while the new Bayes empirical Bayes (BEB) inference approach was used for M2a and M8. Analyses of positive selection by lineage were carried out as previously described (Zhang et al., 2005).

3D modelling. The available structure for the $\mathrm{N}$-terminal, the LRR and IR domains of InlA bound to E-cadherin (pdb code: 1O6S; Schubert et al., 2002) was retrieved from the Protein Data Bank (Berman et al., 2000) and used for analysis and modelling purposes. The structure was used to map nonsynonymous mutations and in particular residues under positive selection.

Invasion assay. Selected isolates, including two isolates with an inlA frameshift mutation (Table 3), were tested for their ability to invade the human intestinal epithelial Caco-2 cell line. Caco-2 invasion assays were performed in 24-well plates essentially as previously described (Nightingale et al., 2005b). Briefly, $1 \mathrm{ml}$ of L. monocytogenes overnight culture (grown at $30{ }^{\circ} \mathrm{C}$ without shaking) was pelleted and resuspended in $1 \mathrm{ml}$ PBS. Confluent Caco-2 monolayers were inoculated with approximately $2 \times 10^{7}$ L. monocytogenes; the inoculum was enumerated on BHI agar plates. Inoculated Caco-2 monolayers were incubated for $30 \mathrm{~min}$ at $37^{\circ} \mathrm{C}$, followed by three washes with PBS and addition of fresh media without antibiotics. Medium containing $150 \mu \mathrm{g}$ gentamicin $\mathrm{ml}^{-1}$ was added $45 \mathrm{~min}$ post-inoculation to kill extracellular bacteria. At 90 min post-inoculation, Caco-2 monolayers were washed three times with PBS and Caco- 2 cells were subsequently lysed by addition of $500 \mu \mathrm{l}$ cold sterile distilled water and vigorous pipetting. Intracellular L. monocytogenes were enumerated by spread plating on BHI agar plates. Each isolate was tested in three different wells on each of three different days (representing three independent replicates). Invasion efficiency was reported as the percentage of inoculum recovered by enumeration of intracellular bacteria. A standard laboratory control strain (10403S) and an isogenic $\Delta i n l A$ strain were included as controls in each invasion assay (Table 2). Data from the three replicates were used for statistical analysis using the Wilcoxon rank sum test (as data were not normally distributed).

Western blot analysis. Western immunoblot analysis was performed as previously described (Nightingale et al., 2005b) to probe for the presence of InlA in bacterial cell wall and supernatant fractions from isolate FSL F2-723, which has an inlA frame shift mutation, and control strain 10403S, which encodes a full-length InlA. Briefly, bacteria were grown in LB supplemented with $50 \mathrm{mM}$ MOPS adjusted to $\mathrm{pH} 7.3,25 \mathrm{mM}$ glucose 1-phosphate and $0.2 \%$ activated charcoal. Chloramphenicol was added to a final concentration of

Table 1. Recombination events in inlA allelic types

A detailed table with all fragments identified by GENECONV or visual evaluation is included as Supplementary Table S3 with the online version of this paper.

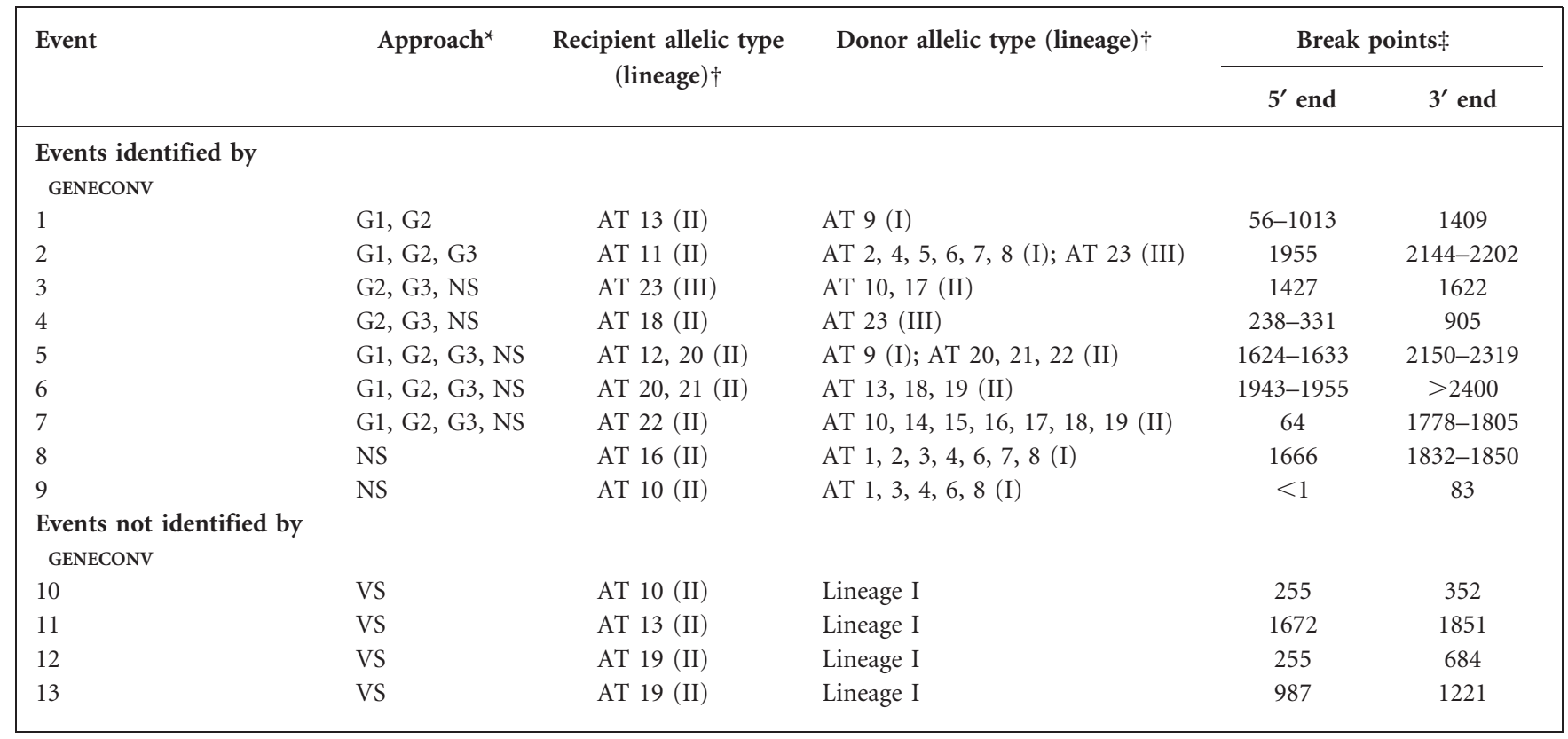

${ }^{\star}$ This column indicates which approach detected a given recombination event; approaches vary in the level and type of mismatches allowed in the recombinant fragment. G1, G2, and G3 represent different G scales in GENECONV; mismatch penalties increase from G1 to G3; NS (no singleton) indicates that a recombination event was detected using an alignment that had all singletons removed and model G0 in GENECONV (which allows for no mismatches); VS (visualization) indicates that recombinant fragments were identified by visual evaluation of polymorphic site alignments. $\dagger$ inlA allelic types (AT) were visually identified as recipient or donor using polymorphic site alignments.

¥Break points shown are from GENECONV outputs (for G1, G2, G3 and NS) or inferred by visualization (for fragments identified by VS); a range of breakpoints is given if GENECONV identified multiple overlapping fragments with different breakpoints (a detailed table with all fragments is provided as Supplementary Table S3). 
Table 2. Summary of positive selection analyses for full-length inlA sequence

\begin{tabular}{|c|c|c|c|c|}
\hline Model $^{\star}$ & $\mathbf{P} \dagger$ & $\ell \ddagger$ & Estimates of parameters $\S$ & Positively selected sites $\|$ \\
\hline M0 & 1 & -5109.03 & $\omega=0.131$ & None \\
\hline M1a & 2 & -5019.55 & $\begin{array}{l}\omega_{0}=0\left(p_{0}=0.905\right) ; \omega_{1}=1 \\
\left(p_{1}=0.095\right)\end{array}$ & Not allowed \\
\hline $\mathrm{M} 2 \mathrm{a}$ & 3 & -5013.18 & $\begin{array}{l}\omega_{0}=0\left(p_{0}=0.938\right) ; \omega_{1}=1 \\
\left(p_{1}=0.0001\right) ; \omega_{2}=\mathbf{2 . 0 6} \\
\left(p_{2}=0.062\right)\end{array}$ & $187^{\mathrm{a}}, 594^{\mathrm{b}}$ \\
\hline M7 & 2 & -5019.62 & $p=0.005 ; q=0.049$ & Not allowed \\
\hline M8 & 4 & -5013.18 & $\begin{array}{l}p=0.837 ; q=99.000 ; \omega=\mathbf{2 . 0 6 3} \\
\left(p_{1}=0.062\right)\end{array}$ & $94^{\mathrm{b}}, 187^{\mathrm{a}}, 594^{\mathrm{a}}, 764^{\mathrm{b}}$ \\
\hline
\end{tabular}

${ }^{*} \mathrm{M}$, model (e.g. M0, model 0); models are detailed in Yang et al. (2000).

$\dagger$ Number of parameters estimated.

\$Likelihood score.

$\S$ For M1a, M2a and M3, $p$ indicates the proportion of sites that fall into a given $\omega$ category; for M7 and M8, $p$ and $q$ are the parameters of the beta distribution; for M8, $p_{1}$ is the proportion of sites that falls into the unconstrained $\omega$ category. All $\omega$ values $>1$ are in bold.

$\|^{\mathrm{b}}$ and ${ }^{\mathrm{a}}$ indicate amino acid sites with posterior probabilities $>0.95$ and $>0.99$, respectively, of being under positive selection; NEB posterior probabilities were calculated for M3 while BEB posterior probabilities were calculated for M2a and M8.

$10 \mathrm{mg} \mathrm{ml}^{-1} 10 \mathrm{~min}$ prior to cell harvest. Cell wall and supernatant fractions were prepared as described previously (Snyder \& Marquis, 2003). Equivalent amounts of culture optical density units were loaded per lane. InlA was detected by Western immunoblotting using a mouse anti-InlA antibody.

Isolate information and nucleotide sequence accession numbers. Isolate information and subtyping data from this study are archived and freely available through the Pathogen Tracker 2.0 database (http://www.pathogentracker.net). inlA sequences have also been deposited in GenBank (accession nos EF445899-EF445938).

\section{RESULTS}

\section{Descriptive analysis}

Sequencing of the full $2400 \mathrm{bp}$ inlA ORF in $40 \mathrm{~L}$. monocytogenes isolates yielded 23 different $i n l A$ allelic types (Supplementary Table S1). Three isolates, including two lineage I, ribotype DUP-1044A isolates (both obtained from human clinical cases) and one lineage II, ribotype DUP-1039C isolate (obtained from a bovine clinical case), presented an in-frame deletion of nine nucleotides (nt 2220-2228), resulting in a sequence of $2391 \mathrm{bp}$. One food isolate (FSL F2-515; lineage II, ribotype DUP-1062A) had a nonsense mutation at nt 2100 that creates a stop codon after aa 699. Another three food isolates (all classified as lineage II, ribotype DUP-1039C) carried a single nucleotide deletion in a homopolymeric tract of seven adenine residues (located at nt 6-12), resulting in a premature stop codon after aa 8 (Fig. 1); all three of these isolates had an identical inlA sequence. With the exception of the frameshift mutation, the inlA sequences for these three isolates were identical to the inlA sequences of other three ribotype DUP-1039C isolates, which had been collected from natural environments. Among the 21 lineage II isolates, 14 had a homopolymeric tract with seven adenines, while four had six adenines with a guanine after the second adenine. All lineage I isolates and the lineage III isolate had a guanine after the second adenine in that region (Fig. 1).

The average $\mathrm{G}+\mathrm{C}$ content for $i n l A$ was $37.3 \mathrm{~mol} \%$. A total of $6.2 \%$ of the inlA sites were polymorphic; these polymorphic sites included 107 sites with synonymous substitutions and 44 sites with nonsynonymous substitutions (representing a total of 45 nonsynonymous changes). The average number of nucleotide differences per site

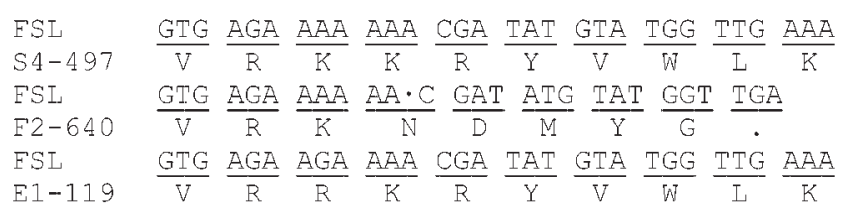

Fig. 1. Single nucleotide deletion at $5^{\prime}$ of inlA that leads to a premature stop codon after aa 8. Isolate FSL S4-497 represents a lineage II isolate with a homopolymeric tract of seven adenines between nt 6 and 12; FSL F2-640 represents a lineage II isolate with deletion of one adenine in the homopolymeric tract; FSL E1119 represents a lineage I isolate with a guanine after the second adenine in the homopolymeric tract (the same sequence was present in all lineage I isolates and the one lineage III isolate characterized). 
between two sequences $(\pi)$ was 0.02134 ; if only nonsynonymous sites were considered, $\pi$ was 0.007 . Lineage II isolates showed a larger inlA nucleotide diversity $\left(\pi_{\mathrm{II}}=0.01322\right)$ than lineage I isolates $\left(\pi_{\mathrm{I}}=0.00526\right)$.

\section{Phylogenetic analysis}

MODELTEST identified the evolutionary model HKY $+\mathrm{G}+\mathrm{I}$ as the most likely model explaining the nucleotide substitution patterns found in the 40 inlA sequences analysed. The molecular clock hypothesis was rejected $(P<0.001)$, indicating that all branches in the tree did not evolve at the same rate. The inlA phylogenetic tree (Fig. 2) constructed using the $\mathrm{HKY}+\mathrm{G}+\mathrm{I}$ model without the molecular clock assumption showed that the inlA sequences represented two distinct clusters, which were consistent with the lineage groupings of isolates based on EcoRI ribotyping; the two main clusters represented lineages I and II (Fig. 2). When phylogenetic trees were generated containing only isolates from lineage I or lineage II, the molecular clock assumption could be rejected for lineage II isolates $(P<0.001)$, but not for lineage I isolates $(P>0.05)$. These results suggest that different evolutionary forces have driven the evolution of inlA of lineage I and

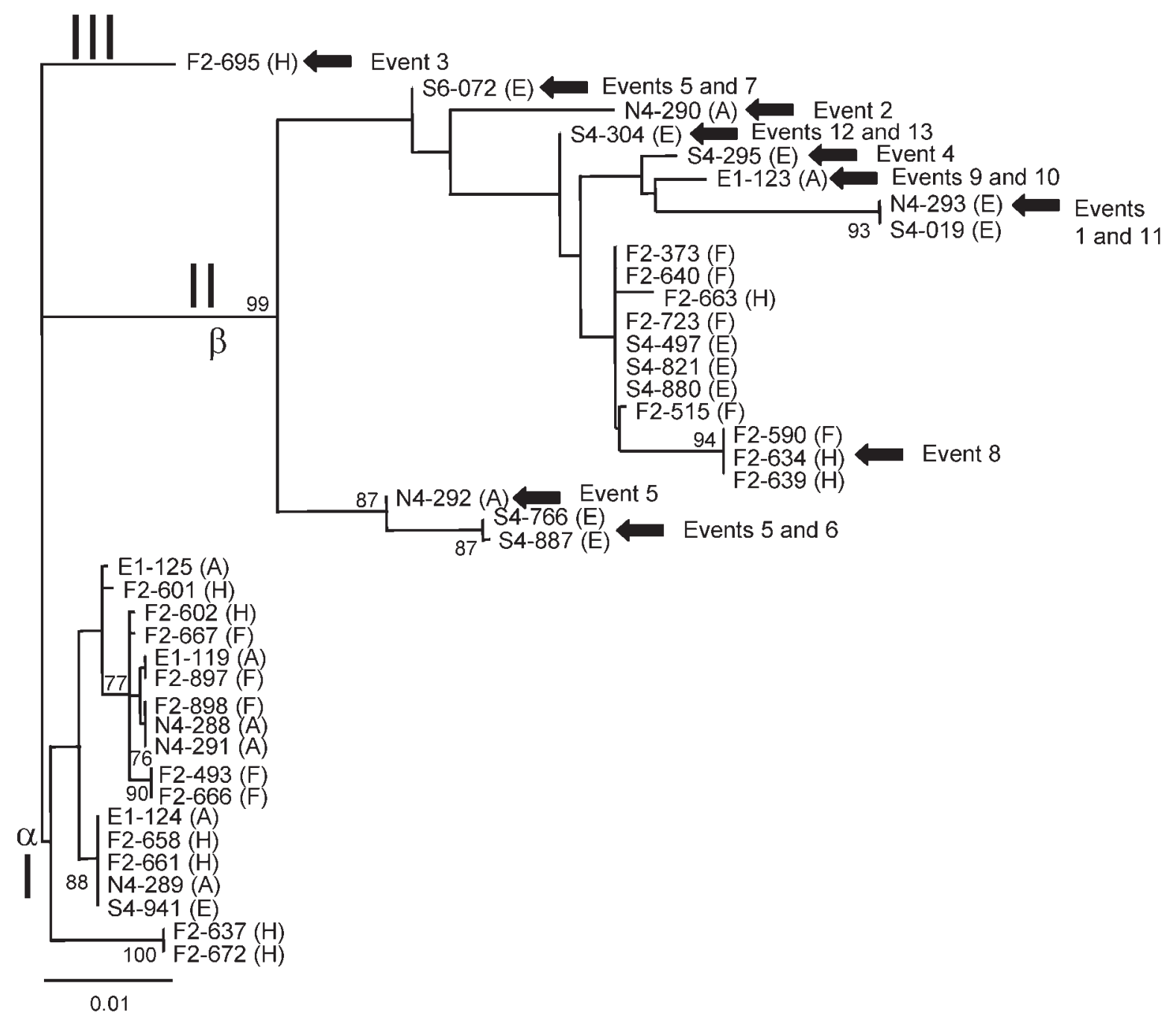

Fig. 2. inlA phylogeny based on 2400 bp nucleotide sequence data for 40 isolates. The tree was constructed using a maximum-likelihood approach and the HKY $+\mathrm{G}+\mathrm{I}$ model without a molecular clock. Isolate FSL F2-695 (lineage III) was set as outgroup. Bootstrap values greater than 50 are shown in the respective branches. Isolates are shown to indicate isolate number (see Supplementary Table S1 for details) and isolate sources, which are shown in parentheses with a one-letter code indicating isolates that were obtained from human clinical cases $(H)$, animals $(A)$, foods $(F)$ or natural environments $(E)$. Arrows indicate sequences in which fragments were introduced by horizontal gene transfer (as determined by GENECONV analysis and/or visually); event numbers are the same as those used in Table 1 and Fig. 3. The symbols $\alpha$ and $\beta$ indicate branches used to test the positive selection for lineage I and lineage II, respectively. 
lineage II isolates and that lineage II inlA is probably exposed to evolutionary forces other than point mutations (e.g. recombination, positive selection).

\section{Recombination analysis}

GENECONV was used to infer possible fragments that do not follow a history of vertical evolution. When the analysis was performed with the GENECONV default setting (G0), which does not allow mismatches within the inferred recombinant fragment, many recombinant fragments had similar breakpoints and involved the same isolates. Visual examination suggested that these fragments represented the same recombination event and that a single recombination event was identified by GENECONV as multiple fragments. Hence, G0 results were not further analysed and GENECONV analysis was repeated using three modifications of the default setting (G1, G2 and G3), which allow for different levels of mismatches within the fragment; the mismatch penalty increases from G1 to G3 and is lowest for G1; mismatch penalties for G2 and G3 are about two and three times higher, respectively, than for G1. G1, G2 and G3 identified a total of 8,58 and 56 global inner fragments, respectively; multiple fragments often showed the same $5^{\prime}$ or $3^{\prime}$ breakpoint, likely indicating that multiple fragments represented a single recombination event (as also described by Nightingale et al., 2005a). Based on visual analyses, all recombinant fragments detected by GENECONV could be classified into seven different recombination events (events 1-7; Table 1). Visual evaluation of an alignment containing only the polymorphic sites identified lineage II isolates as recipients of the recombinant fragment for six events; in one event, the lineage III isolate was identified as recipient. Donors in these seven events included lineage I and II isolates (three events each), as well as the lineage III isolate (one event). Events 5-7 (Table 1; Fig. 3) involved related isolates and appear to represent an ancestral recombination event (event 5, Fig. 3) followed by two other recombination events (events 6 and 7, Fig. 3).

Analysis of the inlA phylogenetic tree (Fig. 2) revealed that seven lineage II inlA sequences (isolates F2-590, F2-634, F2-639, E1-123 and S4-304) and two lineage I sequences (F2-637 and F2-672) that were not identified as recipients in the GENECONV analysis were highly divergent and have long-terminal branches, indicating an accumulation of polymorphisms in these branches. Hence, polymorphic sites alignments for these isolates as well as for all lineage II isolates (due to the large number of long-terminal branches in this lineage) were visually analysed for evidence of additional recombination events. These analyses identified six additional putative recombination events (events 8-13, Table 1), which all appear to have lineage II isolates as recipients and lineage I isolates as donors. To further identify and confirm recombination events, a fourth approach (termed 'no singletons', NS; Table 1) was used; in this approach, all singletons (non-informative sites) in the inlA alignment were removed and GENECONV analysis was performed using the default settings (G0). This procedure is justified because singletons may represent mutations that occurred after the recombination event in either the donor or recipient sequence; this approach differs from G1, G2 and G3 as it specifically removes noninformative sites. This 'NS' procedure identified seven recombination events, including five events previously identified by G1, G2 and G3 (events 3-7, Table 1) and two events previously identified by the visual analysis (events 8 and 9, Table 1, Fig. 4). In summary, we identified a total of 13 inlA recombination events, 12 of them involving lineage II isolates as recipients. Fourteen out of the 21 lineage II isolates showed evidence for at least one horizontal gene transfer event, including 4/4 animal, 6/9 environmental, $2 / 3$ human and $1 / 5$ food isolates classified in lineage II. While we detected 13 recombination events, it is feasible that some recombination events (e.g. between closely related sequences in lineage I) may not have been detected, and that additional recombination events were involved in the evolution of the inlA sequences studied here.

\section{Positive selection in inlA}

Nested models of heterogeneous codon substitution (Yang et al., 2000, 2005) were used to determine whether inlA has evolved by positive selection. In these models, $\omega$ represents the $d_{\mathrm{N}} / d_{\mathrm{S}}$ ratio [i.e. (no. of nonsynonymous changes/no. of nonsynonymous sites) /(no. of synonymous changes/no. of synonymous sites)]; $\omega>1$ is indicative of positive selection, $\omega=1$ is indicative of neutrality and $\omega<1$ is indicative of purifying selection. The comparison between M0 and M3 may be interpreted as a test for variability in the $\omega$ ratio

Table 3. L. monocytogenes strains and isolates used for invasion assays (IA) and Western blot (WB)

\begin{tabular}{|llll|}
\hline Strain/isolate & \multicolumn{1}{c}{ Relevant strain/isolate characteristics } & Experiment & Source or reference \\
\hline $10403 S$ & Laboratory control strain & IA \& WB & Bishop \& Hinrichs (1987) \\
FSL K4-006 & 10403S $\Delta$ inlA (DP-L4405) & IA \& WB & $\begin{array}{l}\text { Bakardjiev et al. (2004) } \\
\text { This article }\end{array}$ \\
FSL S4-497 & Environmental isolate, ribotype DUP-1039C, full-length inlA & IA & This article \\
FSL F2-723 & $\begin{array}{l}\text { Food isolate, ribotype DUP-1039C, inlA frameshift mutation } \\
\text { resulting in a stop codon after aa 8 }\end{array}$ & IA \& WB & This article \\
FSL F2-640 & $\begin{array}{l}\text { Food isolate, ribotype DUP-1039C, inlA frameshift mutation } \\
\text { resulting in a stop codon after aa 8 }\end{array}$ & IA & \\
\hline
\end{tabular}




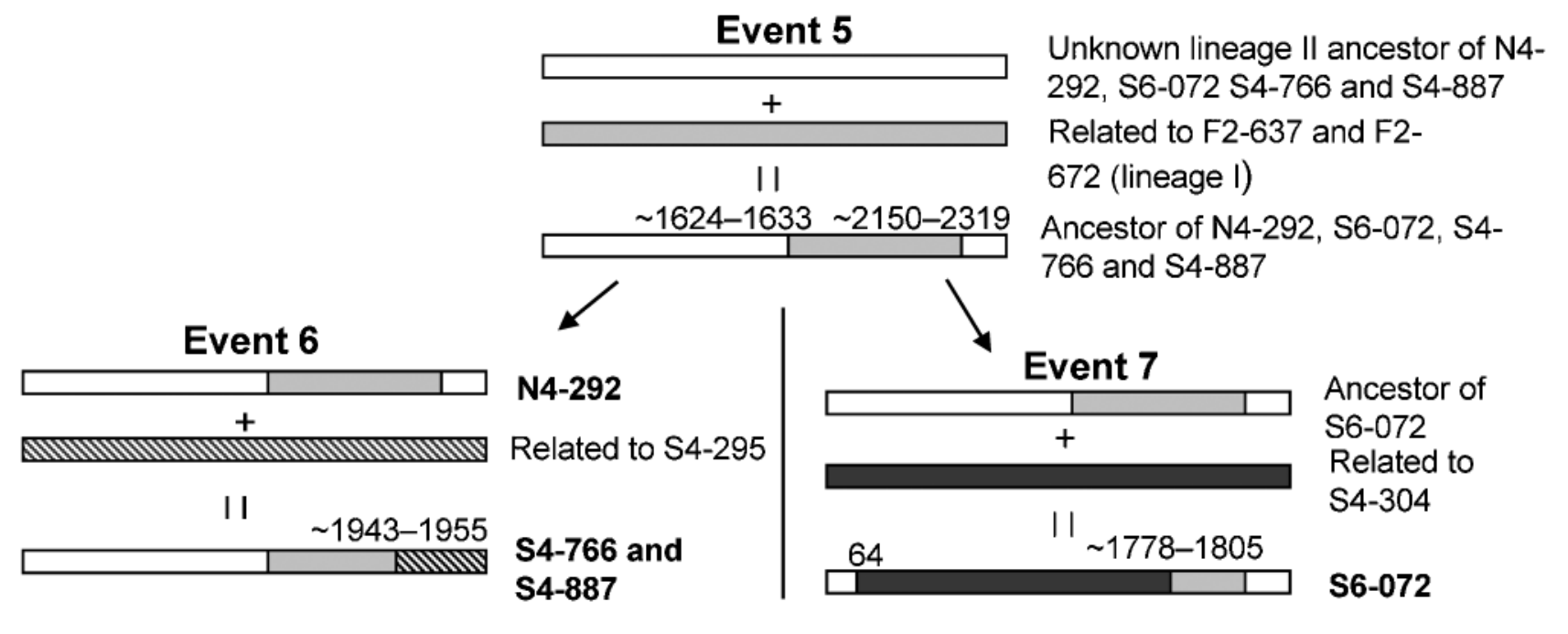

Fig. 3. Schematic representation of recombination events 5,6 and 7 . The grey sequence is a lineage I sequence that is most closely related to the inlA sequence for isolates FSL F2-637 and FSL F2-672 (inIA AT 9). The white sequence represents a lineage II inlA sequence likely representing the ancestor of the inlA sequences in lineage II isolates FSL N4-292, FSL S6-072, FSL S4-766 and FSL S4-887. The hatched sequence represents a lineage II inlA sequence most closely related to the inlA sequences for isolate FSL S4-295. The black sequence represents a lineage II inlA sequence most closely related to the inlA sequences for isolates FSL S4-304. Bold isolate names identify inlA sequences for isolates that were sequenced as part of this study. Numbers above sequences indicate break points; the $3^{\prime}$ break point for event 6 is located $3^{\prime}$ of the sequenced fragment and is thus not indicated.

among sites (Yang \& Nielsen, 2002). M0 was rejected in favour of M3 $(P<0.001)$, indicating that the assumption of a single $\omega$ for all sites along a sequence is not valid and that $\omega$ varies along the length of the inlA sequence.

Four models were used to determine whether positive selection is likely to have occurred in inlA. Model 1a (M1a) is a neutral model, which assumes two classes of $\omega$, including (i) $\omega_{0} \quad\left(0<\omega_{0}<1\right.$; representing codons under negative selection) and (ii) $\omega_{1}\left(\omega_{1}=1\right.$; representing codons under neutral selection). Model 2a (M2a) allows for a third class of $\omega\left(\omega_{2}>1\right.$; representing codons under positive selection). Model 7 (M7) uses a discrete approximation of a beta distribution and does not allow a site class where $\omega>1$; model 8 (M8) allows for an additional site class where $\omega>1$. M1a was rejected in favour of M2a $(P<0.005)$ and M7 was rejected in favour of M8 $(P<0.005)$, indicating that positive selection has likely played a role in the evolution of inlA. M2a identified two sites with posterior probabilities of being under positive selection greater than $95 \%$, while M8 identified four sites, which included the two sites found by M2a (Table 2).

Model A as described by Zhang et al. (2005) was used to test the hypothesis that positive selection has occurred in specific lineages (lineage I or lineage II). Test 2 compares model A, which allows for positive selection along one specific branch or set of branches, and model A null, which does not allow for positive selection along the same branch or set of branches (Zhang et al., 2005). Each lineage was tested independently (see Fig. 2; branches tested by these models are indicated by Greek letters). First, the branch that originated each lineage was tested using the models and then all the branches that belong to each lineage were also tested. We found no evidence for positive selection in lineage I. Conversely, when all branches derived from branch $\beta$ were tested (meaning all lineage II branches), model A null could be rejected $(P<0.001)$, indicating positive selection restricted to lineage II isolates. Model A identified a single site with BEB posterior probability $>0.95$ as being under positive selection in linage II (site 594; $P=0.989$ ); this site was also identified as being under positive selection by M2a and M8.

As we were aware that the $d_{\mathrm{N}} / d_{\mathrm{S}}$ tests implemented in PAML assume no recombination and knowledge of the true phylogeny (Anisimova et al., 2003; Nielsen, 2001), we repeated the analysis using an alignment without the inlA sequences that were identified to be recipients of fragments by horizontal gene transfer. In this analysis, we could not reject any of the null models (M0, M1a, M7) in favour of the alternative models (M3, M2a and M8) and no sites were identified to be under positive selection. However, 16 of the 45 nonsynonymous substitutions were found only in the 14 isolates that showed evidence for recombination in inlA. By comparison, eight nonsynonymous substitutions were only found in the 26 isolates without evidence for recombination in inlA; 21 nonsynonymous substitutions were shared between these two groups of isolates. While our analyses thus may indicate that recombination events may have affected our positive selection analyses, the observation 
(a)

000000000000000000000000000000000000000111111111111111111111

00000001112222222333344445556667999999900011111222222222333

056689915511234583345266811626880445788133344692234456791245

850343560139379500622459616010406264257258439711832786734310

e1124 GTCTTAACTAGACGGATTCTGT GATCTGAGATGACAATACGGATCGTTTACACGTAGGT

e1125

f2897

f2667

f2898

f2601

f2602

f2590

f2663

S4497
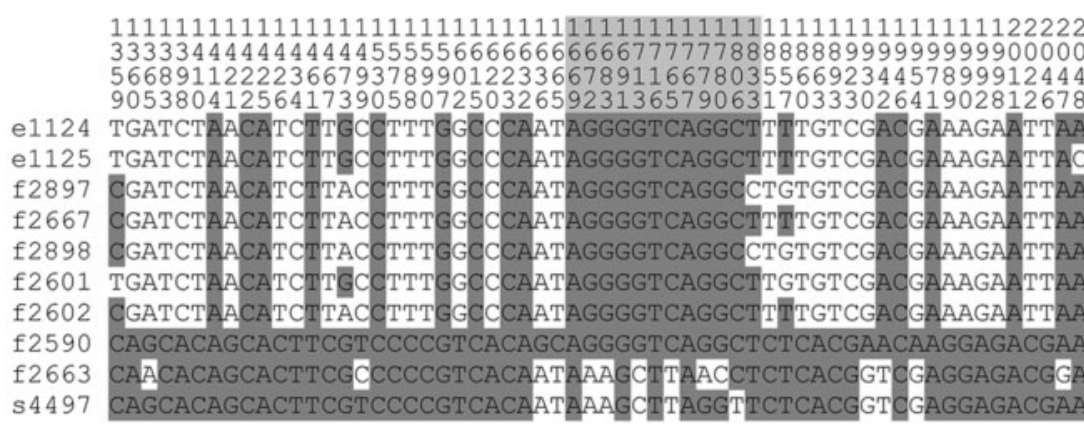

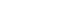

\begin{abstract}
2222222222222222222222222222
0001111111111122222233333333

6880012455677803499901234446

7580997517028332412443071698
\end{abstract}

e1124 ATTCCCAGTGTACCCTTAACTT GAAGG
e1125 ATTCCCAGTGTACCCTTAACTTGGAAG

e1125

f2667

f2898

f2601

f2602

f2590

f2663

s4497 (b)

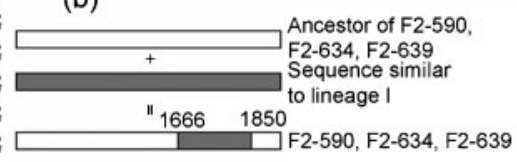

Fig. 4. Recombination event 8 , which is responsible for generation of the inlA sequence found in isolates FSL F2-590, FSL F2-634 and FSL F2-639. (a) Alignment of the polymorphic sites of the inlA sequences identified by GENECONV as being involved in this event. FSL S4-497 (labelled as s4497) and FSL F2-663 (labelled as f2663) are lineage II isolates; inlA sequences for these isolates are more closely related to the $\mathrm{in} / A$ sequence for FSL F2-590 (a lineage II strain, as determined by ribotyping) than the $i n / A$ sequences for the seven lineage $I$ isolates included (labelled as e1124, e1125, f2897, f2667, f2898, f2601 and f2602). Shaded areas are nucleotides that are identical to the F2-590 sequence. Numbers above the alignment represent the positions of the polymorphic sites in the alignment. (b) Schematic representation of recombination event 8; nucleotide sites above the sequence represent the location of the $5^{\prime}$ and $3^{\prime}$ breakpoints. that no evidence of positive selection was found when analyses were performed only on 26 inlA sequences without any apparent history of recombination may also reflect reduced power of these analyses as this dataset contains a considerably smaller number of nonsynonymous substitutions as compared to all 40 inlA sequences.

\section{Positive selection by functional InIA regions}

InlA can be divided into eight regions, which have distinct characteristics and functions. The LRR was found to be the most conserved region (only $1.4 \%$ of amino acid sites are polymorphic), while PGS is the most variable region, with $10 \%$ of amino acid sites being variable (Fig. 5). To determine how selection acts on these eight regions, the $d_{\mathrm{N}} /$ $d_{\mathrm{S}}$ overall ratio was calculated for each region. Nested models of evolution, including (i) M0 and M3, (ii) M1a and M2a and (iii) M7 and M8 were also analysed as described in detail above. The signal peptide (SP) and interdomain (ID) regions were not analysed because the first is not present in the mature InlA and the second is too short (23 aa) to provide reliable estimates of parameters. For two regions (i.e. the LRR and B-repeat region), M0 was rejected and M3 was accepted. M1a could not be rejected for any region $(P>0.05)$, even though for the LRR region, M8 was accepted over M7, providing evidence for positive selection in the LRR region. One site (codon 187) was identified by M8 as being under positive selection in the LRR region (Fig. 5); this site had also been identified as being under positive selection by M2a and M8 using the complete inlA sequence (Table 2). Consistent with the fact that M3 was accepted when the whole sequence was analysed, these results suggest that the different regions of InlA are under different selective pressures.

\section{Mapping of the polymorphic and positively selected sites using the available crystallized structure of InIA}

The partial crystallized structure of InlA, which includes the LRR region as well as the $\alpha$-helical and the Ig-like domains (Schubert et al., 2002), was used to map the localization of the polymorphic amino acid sites as well as positively selected sites identified by M8 (see above). Among the 44 polymorphic amino acids sites (including the four positively selected sites, which were identified by 


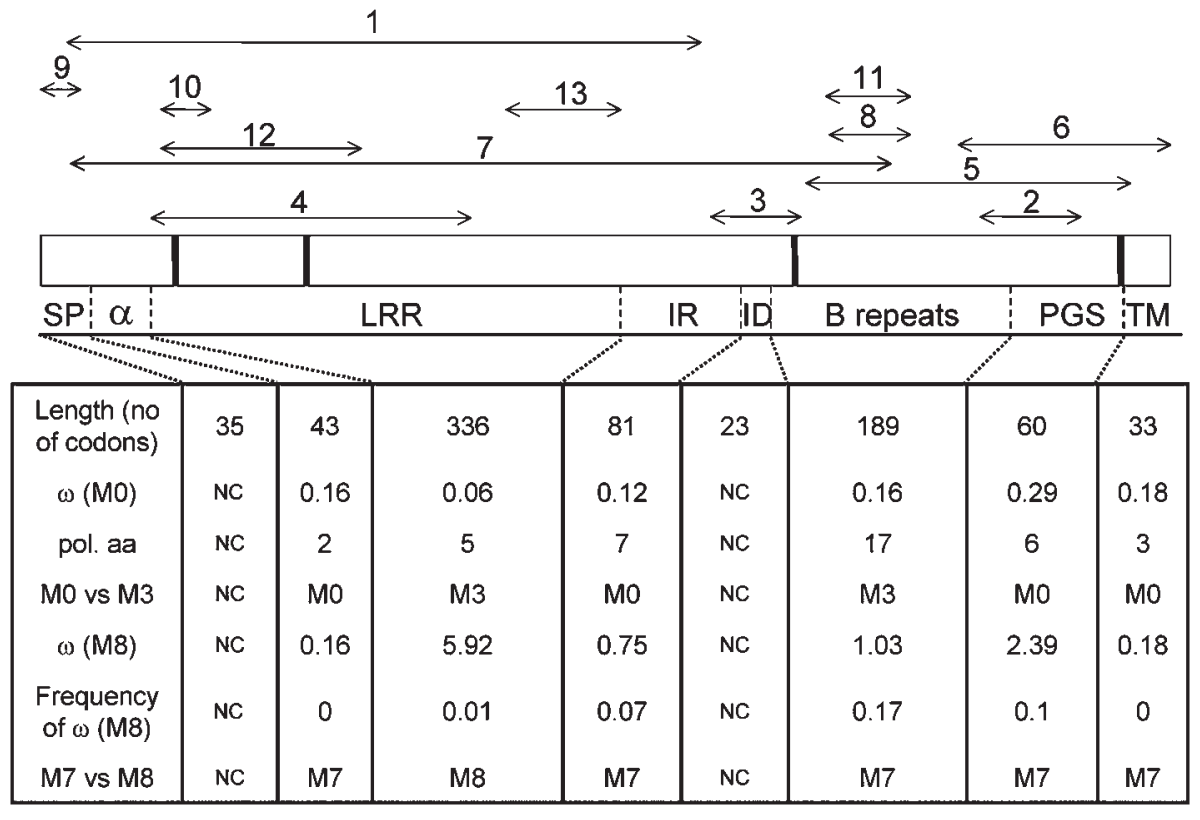

Fig. 5. Schematic representation of InIA with recombination events and positively selected sites. Recombinant fragments (113; numbers are identical to those used in Table 1) are shown above the structure. The functional regions are shown below the structure: SP, signal peptide; $\alpha, \alpha$-helix domain; LRR, leucine-rich repeat; IR, inter-region; ID, interdomain; PGS, proline/glycinerich segment; TM, transmembrane domain. Positively selected sites identified by M8 are represented as vertical lines (the codon for aa 187 was identified by M2a and M8 performed on the full-length inlA sequences as well as by M8 performed on the LRR regions only). The table lists the $\omega\left(d_{N} / d_{S}\right)$ value for each region (as determined by $\mathrm{MO}$ ) as well as the number of polymorphic amino acids in a given region (pol. aa). The results of comparisons between M0 and M3 (M0 vs M3) and M7 and M8 (M7 vs M8) are also shown; the model that best describes the substitution patterns for a given region is indicated (e.g. M8 indicates that $M 8$ was accepted). ' $\omega$ (M8)' indicates the $\omega$ value for sites classified by M8 into the site category with $\omega>1$; 'frequency of $\omega$ (M8)' indicates the frequency of sites classified by M8 in the site category with $\omega>1$. NC indicates regions for which values were not calculated.

M8), 14 polymorphic amino acids sites (including two sites under positive selection) were located in the InlA regions that are included in the partial crystallized structure (Fig. 6). All positively selected amino acid were found to be located on the outer surface of the LRR domain and no polymorphic amino acids were identified in the inner surface of the LRR domain, including the specific region that interacts with E-cadherin.

\section{Invasiveness of isolates with an inIA frameshift mutation}

Invasion assays on two representative ribotype DUP-1039C isolates with a premature stop codon after InlA aa 8 were carried out to investigate the invasiveness of these isolates. The two isolates bearing the frameshift mutation were significantly $(P<0.05$; invasion efficiencies of 0.010 and $0.013 \%$ for isolates FSL F2-723 and FSL F2-640, respectively) less invasive than strain 10403S (a laboratory control strain that encodes a full-length InlA; invasion efficiency of $0.139 \%$ ) and isolate FSL S4-497 (a ribotype DUP1039C isolate that encodes a full-length InlA; invasion efficiency of $1.48 \%$ ). The two isolates with the inlA frameshift mutation did not differ significantly $(P>0.05)$ in their invasiveness from strain FSL K4-006, a $\Delta$ inlA mutant derived from strain $10403 \mathrm{~S}$ (invasion efficiency of $0.004 \%)$.

\section{Western blot analysis of a selected isolate with an inIA frameshift mutation}

Western blot analysis of the cytoplasmic protein fraction and culture supernatant of an isolate with an inlA frameshift mutation (i.e. isolate FSL F2-723) was performed to confirm the premature stop codon after InlA aa 8 identified in three isolates based on inlA nucleotide sequence data. While no InlA was detected in the cell wall (not shown) and supernatant fraction of isolate FSL F2-723 (Fig. 7), a weak band with the expected size for InlA was detected in the cytoplasmic extract of isolate FSL F2-723 (Fig. 7). We hypothesized that this weak band is due to weak translation of InlA from a second potential start codon [located at nt 169-171, which encodes aa 57 (methionine) in the full-length InlA]. This hypothesis would also explain why InlA was not identified in the cell wall or supernatant, since this potential start codon is just 


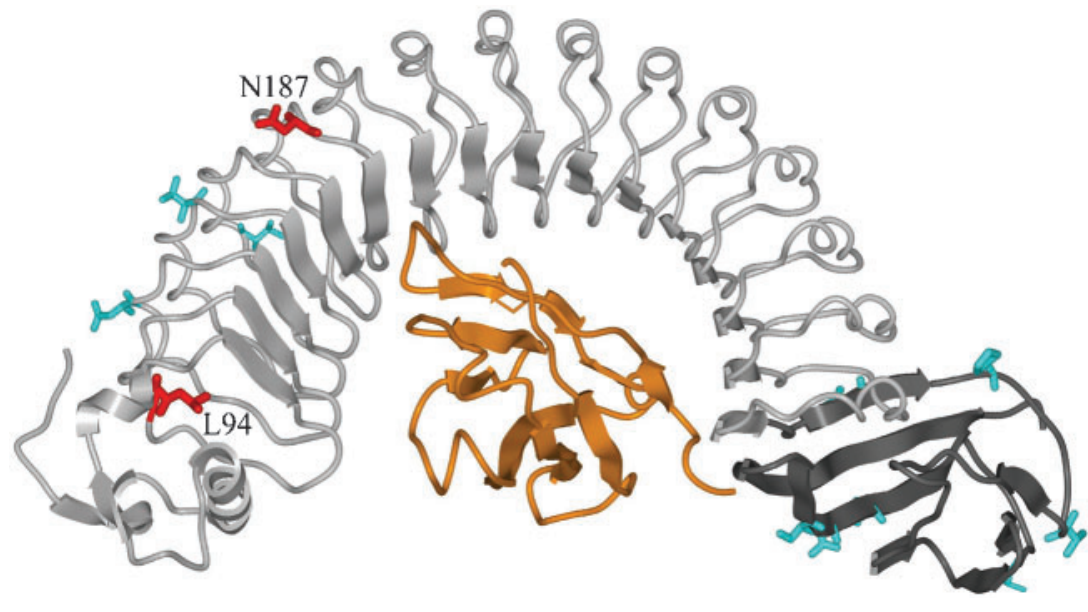

Fig. 6. Partial structure of $\ln \mid \mathrm{A}$ (grey) in contact with the human receptor E-cadherin (orange). The two sites with posterior probabilities $>95 \%$ of being under positive selection (i.e. sites 94 and 187) are shown in red; the two other sites with posterior probabilities $>95 \%$ of being under positive selection (i.e. sites 594 and 764) are located outside the region encompassed by this structure. Polymorphic sites that are not under positive selection are shown in light blue. downstream of the signal peptide region, which would not be present in a truncated protein translated from this start codon.

\section{DISCUSSION}

Overall, our data show that (i) inlA is highly polymorphic and evolution of inlA involved a considerable number of recombination events in lineage II isolates; (ii) positive selection at specific amino acid sites appears to contribute to evolution of inlA, including fixation of recombinant events; and (iii) a single nucleotide deletion in a lineage IIspecific $5^{\prime}$ homopolymeric tract in inlA leads to complete loss of InlA or to production of truncated InlA, which is associated with reduced invasiveness. The identification of both polymorphic and positively selected sites in inlA as reported here also provides an opportunity for future studies that include generation and characterization of

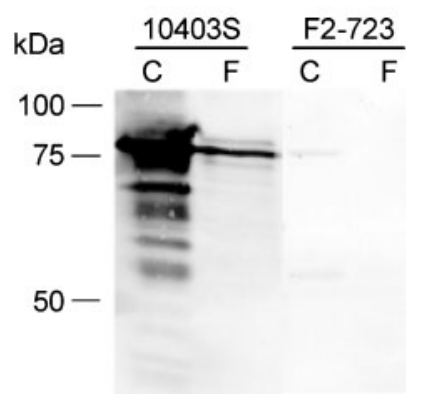

Fig. 7. $\operatorname{In} \mid A$ Western blot to determine $\operatorname{In} \mid A$ presence in a $L$. monocytogenes isolate with premature stop codons after $\operatorname{InI} \mathrm{A}$ aa 8 . Western blot was performed with isolate FSL F2-723 (which has a premature InIA stop codon) and strain 10403S, which encodes a full-length InIA (Table 3). Western blot analysis was performed on cytoplasmic protein fractions (C) and on proteins precipitated from culture supernatants (S). The expected molecular mass of $\operatorname{In} \mid \mathrm{A}$ is approximately $80 \mathrm{kDa}$. isogenic strains carrying different inlA allelic variants to explore the phenotypic consequences of these mutations.

\section{inlA is highly polymorphic and evolution of inIA involved a considerable number of recombination events in lineage II isolates}

Overall nucleotide diversity $(\pi=0.02134)$ as well as the number of nonsynonymous sites were high for inlA, but were within the range previously described for six other internalin genes (inlB, inlC2, inlD, inlE, inlF and inlG) that had previously been sequenced in the same 40 isolates characterized here (Tsai et al., 2006). Thus, this group of $L$. monocytogenes surface proteins is characterized by considerable overall nucleotide and amino acid diversity. Similar to the observation that all of the six other $L$. monocytogenes internalins previously characterized by Tsai et al. (2006) showed evidence for recombination, we have identified a number of recombination events among the 23 inlA allelic types identified here. Interestingly, all but one of the inlA recombination events identified involved lineage II strains as recipients. This is consistent with previous observations that lineage II strains show evidence for considerably more horizontal gene transfer than lineage I isolates (Meinersmann et al., 2004; Nightingale et al., 2005a) and that most recombination events in other internalin genes involved lineage II strains as recipients (Tsai et al., 2006). The important role for recombination in generating diversity in selected L. monocytogenes genes is further supported by Nightingale et al. (2005a), who showed that L. monocytogenes genes with weak (i.e. prs) or no evidence for recombination (i.e. gap and $\operatorname{sig} B$ ) showed lower diversity than housekeeping genes with strong evidence for recombination (i.e. purM, ribC). Our data are also consistent with a number of studies that have shown that recombination plays an important role in the diversification of genes encoding surface proteins in many pathogens, including the Escherichia coli intimin gene (McGraw et al., 1999) and fimA (Peek et al., 2001), the Plasmodium falciparum AMA1 gene (Polley \& Conway, 
2001) as well as Neisseria meningitidis pilE (Andrews \& Gojobori, 2004) and porB (Urwin et al., 2002). Recombination within genes encoding surface proteins, including inlA, may specifically be important by providing rapid diversification that allows for evasion of the host immunity system and for adaptive shifts in host or tissue tropism (Andrews \& Gojobori, 2004; McGraw et al., 1999; Peek et al., 2001; Polley \& Conway, 2001; Urwin et al., 2002).

\section{Positive selection at specific amino acid sites appears to contribute to evolution of inIA, including fixation of recombinant events}

Our analysis indicated that specific amino acid sites in inlA are under positive selection and that positive selection appears to specifically act on lineage II inlA. While we are aware that our dataset violated one of the assumptions of the positive selection analysis used (i.e. absence of recombination) (Anisimova et al., 2003), there are currently no methods available that can be used to test for evidence of positive selection in genes that have a history of recombination. While recombination, particularly in early internal branches, can affect the reliability of the LRT (Anisimova et al., 2003), Bayes' identification of positively selected sites seems to be less sensitive to the tree topology (Anisimova et al., 2003), and therefore can be used even when recombination is present. As Bayes' identification of positively selected sites found a number of positively selected sites, we are confident that our results correctly suggest that inlA has undergone positive selection. We specifically hypothesize that positive selection may contribute to fixation of recombinant inlA genes that encode for proteins that provide for increased fitness in the L. monocytogenes population sampled. This is consistent with the hypothesis that, in order to maintain recombinant fragments in a population, such fragments should be advantageous to the organism (Andrews \& Gojobori, 2004; Milkman et al., 2003). Recombination has previously been suggested to act together with positive selection in several genes in different organisms (Andrews \& Gojobori, 2004; Peek et al., 2001; Polley \& Conway, 2001; Urwin et al., 2002), including many pathogens (Andrews \& Gojobori, 2004; Milkman et al., 2003; Polley \& Conway, 2001). For example, strong positive selection and recombination seem to drive the antigenic variation of the PilE protein of the human pathogen N. meningitidis (Andrews \& Gojobori, 2004). In E. coli, recombination in fimA has been proposed to allow for diversifying and purifying selection to occur in different regions of the same gene by uncoupling these regions (Peek et al., 2001). Similar mechanisms may be at work in inlA, where one area, which contacts the human receptor E-cadherin, seems to be under strong purifying selection while other regions may be under diversifying selection, possibly in response to selective pressure imposed by the host immune response, which may select for changes in epitopes found on the outer surface of pathogen proteins (Andrews \& Gojobori, 2004; Peek et al.,
2001; Polley \& Conway, 2001). While this is consistent with the observation that both of the positively selected sites that could be mapped to the InlA crystal structure were totally or partially exposed on the surface of InlA, further studies are necessary to determine whether these sites are indeed recognized by the host immune system.

\section{A single nucleotide deletion in a lineage II- specific 5' homopolymeric tract in inlA leads to complete loss of InIA or to production of truncated InIA, which is associated with reduced invasiveness}

Among the 40 isolates characterized here, we identified four food isolates that carried mutations in inlA that led either to a premature stop codon after aa 699 (one isolate) or to a premature stop codon after aa 8 (three isolates). The inlA allelic type encoding a premature stop codon after aa 699 has previously been reported by Nightingale et al. (2005b) and leads to expression of a truncated and secreted form of InlA that lacks the cell-wall-binding LPXTG motif; this premature stop codon appears to be common among food isolates in the USA (Nightingale et al., 2005b). However, the frameshift mutation that leads to an early premature stop codon (after aa 8) has, to our knowledge, not previously been described. Interestingly, the mutations leading to this premature stop codon represents a single nucleotide deletion in a homopolymeric tract of seven adenines, which is found in 14/21 lineage II inlA sequences. Frameshift mutations due to single nucleotide deletions on homopolymeric tracts have previously been reported to occur naturally in a number of organisms (e.g. Kearns et al., 2004; Segura et al., 2004; Theiss \& Wise, 1997) and these types of deletion events appear to be reversible, allowing organisms to undergo phase variation. For example, phase variation involving a frameshift mutation in a run of $\mathrm{A}: \mathrm{T}$ base pairs has been reported in a swarming gene in Bacillus subtilis (Kearns et al., 2004). Future experiments will thus focus on evaluating the reversibility of the inlA frameshift mutation identified here. As we have identified identical inlA sequences with and without the single adenine deletion that leads to a premature stop codon, we hypothesize that this mutation may be reversible, possibly allowing for InlA phase variation. Interestingly, while most lineage II strains, which are overrepresented among food and environmental isolates and underrepresented among human isolates (Sauders et al., 2006), have a homopolymeric tract of seven adenines, this homopolymeric tract was found here to be interrupted by a guanine in strains that group into lineage I, a lineage which appears to be overrepresented among human isolates (Sauders et al., 2006). We hypothesize that potentially environmentally adapted $L$. monocytogenes (i.e. many lineage II strains) may have evolved to allow for InlA phase variation, while potentially human-adapted strains may have evolved to stably express InlA. Interestingly, our recombination analyses showed that at least one of the four lineage II isolates with a homopolymeric tract of seven adenines at 
the $5^{\prime}$ end of inlA that is interrupted by a guanine acquired this inlA fragment by horizontal gene transfer (event 9) from a lineage I strain, indicating that some lineage II strains may evolve towards stable expression of full-length InlA.

Our data also provide further evidence that mutations leading to premature stop codons in inlA occur commonly and at different locations in the genes, leading to either expression of a secreted InlA or to expression of truncated proteins, which are unlikely to be functional. Specifically, characterization of L. monocytogenes isolates from France (Jonquieres et al., 1998; Olier et al., 2002, 2003; Rousseaux et al., 2004) and the USA (this study, Nightingale et al., 2005b) so far have identified at least 10 distinct mutations leading to premature stop codons in inlA. A number of the mutations that occur in different sites in the $5^{\prime}$ end of inlA have previously been shown to be associated with a decreased ability of L. monocytogenes to invade epithelial cells (Olier et al., 2003; Nightingale et al., 2005b). It thus seems that there is a strong selective force driving the loss of a functional InlA in some L. monocytogenes isolates, particularly those found in foods, as supported by the fact that a considerable number of independent mutations leading to a loss of functional, cell-wall-bound InlA have been found and appear to have been fixed in the populations studied. Thus, lack of a cell-wall-bound InlA or complete abolition of InlA expression may provide a selective advantage for certain L. monocytogenes subtypes in certain environments. The specific selection pressure responsible for this remains to be determined though, which is likely to prove challenging considering that $L$. monocytogenes is found in a diversity of environments and can infect a number of mammalian and non-mammalian host species (Mansfield et al., 2003; Sauders et al., 2006; Vazquez-Boland et al., 2001).

\section{ACKNOWLEDGEMENTS}

This work was supported by a USDA Special Research Grant (200534459-15625). We thank Qi Sun for helpful discussions. This research was conducted in part by using the resources of the Cornell Theory Center, which receives funding from Cornell University, New York State, federal agencies, foundations, and corporate partners.

\section{REFERENCES}

Andrews, T. D. \& Gojobori, T. (2004). Strong positive selection and recombination drive the antigenic variation of the PilE protein of the human pathogen Neisseria meningitidis. Genetics 166, 25-32.

Anisimova, M., Nielsen, R. \& Yang, Z. (2003). Effect of recombination on the accuracy of the likelihood method for detecting positive selection at amino acid sites. Genetics 164, 1229-1236.

Bakardjiev, A. I., Stacy, B. A., Fisher, S. J. \& Portnoy, D. A. (2004). Listeriosis in the pregnant guinea pig: a model of vertical transmission. Infect Immun 72, 489-497.

Berman, H. M., Bhat, T. N., Bourne, P. E., Feng, Z., Gilliland, G. Weissig, H. \& Westbrook, J. (2000). The Protein Data Bank and the challenge of structural genomics. Nat Struct Biol 7 (Suppl.), 957-959.
Bishop, D. K. \& Hinrichs, D. J. (1987). Adoptive transfer of immunity to Listeria monocytogenes. The influence of in vitro stimulation on lymphocyte subset requirements. J Immunol 139, 2005-2009.

Dhar, G., Faull, K. F. \& Schneewind, O. (2000). Anchor structure of cell wall surface proteins in Listeria monocytogenes. Biochemistry 39, 3725-3733.

Dramsi, S., Kocks, C., Forestier, C. \& Cossart, P. (1993). Internalinmediated invasion of epithelial cells by Listeria monocytogenes is regulated by the bacterial growth state, temperature and the pleiotropic activator prfA. Mol Microbiol 9, 931-941.

Gaillard, J. L., Berche, P., Frehel, C., Gouin, E. \& Cossart, P. (1991). Entry of L. monocytogenes into cells is mediated by internalin, a repeat protein reminiscent of surface antigens from gram-positive cocci. Cell 65, 1127-1141.

Gray, M. J., Zadoks, R. N., Fortes, E. D., Dogan, B., Cai, S., Chen, Y., Scott, V. N., Gombas, D. E., Boor, K. J. \& Wiedmann, M. (2004). Listeria monocytogenes isolates from foods and humans form distinct but overlapping populations. Appl Environ Microbiol 70, 5833-5841.

Jeffers, G. T., Bruce, J. L., McDonough, P. L., Scarlett, J., Boor, K. J. \& Wiedmann, M. (2001). Comparative genetic characterization of Listeria monocytogenes isolates from human and animal listeriosis cases. Microbiology 147, 1095-1104.

Jonquieres, R., Bierne, H., Mengaud, J. \& Cossart, P. (1998). The inlA gene of Listeria monocytogenes LO28 harbors a nonsense mutation resulting in release of internalin. Infect Immun 66, 3420-3422.

Kearns, D. B., Chu, F., Rudner, R. \& Losick, R. (2004). Genes governing swarming in Bacillus subtilis and evidence for a phase variation mechanism controlling surface motility. Mol Microbiol 52, 357-369.

Lecuit, M., Dramsi, S., Gottardi, C., Fedor-Chaiken, M., Gumbiner, B. \& Cossart, P. (1999). A single amino acid in E-cadherin responsible for host specificity towards the human pathogen Listeria monocytogenes. EMBO J 18, 3956-3963.

Lecuit, M., Vandormael-Pournin, S., Lefort, J., Huerre, M., Gounon, P., Dupuy, C., Babinet, C. \& Cossart, P. (2001). A transgenic model for listeriosis: role of internalin in crossing the intestinal barrier. Science 292, 1722-1725.

Lecuit, M., Nelson, D. M., Smith, S. D., Khun, H., Huerre, M., VacherLavenu, M. C., Gordon, J. I. \& Cossart, P. (2004). Targeting and crossing of the human maternofetal barrier by Listeria monocytogenes: role of internalin interaction with trophoblast E-cadherin. Proc Natl Acad Sci U S A 101, 6152-6157.

Mansfield, B. E., Dionne, M. S., Schneider, D. S. \& Freitag, N. E. (2003). Exploration of host-pathogen interactions using Listeria monocytogenes and Drosophila melanogaster. Cell Microbiol 5, 901-911.

McGraw, E. A., Li, J., Selander, R. K. \& Whittam, T. S. (1999). Molecular evolution and mosaic structure of $\alpha, \beta$, and $\gamma$ intimins of pathogenic Escherichia coli. Mol Biol Evol 16, 12-22.

Meinersmann, R. J., Phillips, R. W., Wiedmann, M. \& Berrang, M. E. (2004). Multilocus sequence typing of Listeria monocytogenes by use of hypervariable genes reveals clonal and recombination histories of three lineages. Appl Environ Microbiol 70, 2193-2203.

Mengaud, J., Lecuit, M., Lebrun, M., Nato, F., Mazie, J. C. \& Cossart, P. (1996). Antibodies to the leucine-rich repeat region of internalin block entry of Listeria monocytogenes into cells expressing E-cadherin. Infect Immun 64, 5430-5433.

Milkman, R., Jaeger, E. \& McBride, R. D. (2003). Molecular evolution of the Escherichia coli chromosome. VI. Two regions of high effective recombination. Genetics 163, 475-483.

Nielsen, R. (2001). Statistical tests of selective neutrality in the age of genomics. Heredity 86, 641-647. 
Nightingale, K. K., Windham, K. \& Wiedmann, M. (2005a). Evolution and molecular phylogeny of Listeria monocytogenes isolated from human and animal listeriosis cases and foods. J Bacteriol 187, 5537-5551.

Nightingale, K. K., Windham, K., Martin, K. E., Yeung, M. \& Wiedmann, M. (2005b). Select Listeria monocytogenes subtypes commonly found in foods carry distinct nonsense mutations in inlA, leading to expression of truncated and secreted internalin $\mathrm{A}$, and are associated with a reduced invasion phenotype for human intestinal epithelial cells. Appl Environ Microbiol 71, 8764-8772.

Olier, M., Pierre, F., Lemaitre, J. P., Divies, C., Rousset, A. \& Guzzo, J. (2002). Assessment of the pathogenic potential of two Listeria monocytogenes human faecal carriage isolates. Microbiology 148, $1855-1862$.

Olier, M., Pierre, F., Rousseaux, S., Lemaitre, J. P., Rousset, A., Piveteau, P. \& Guzzo, J. (2003). Expression of truncated internalin A is involved in impaired internalization of some Listeria monocytogenes isolates carried asymptomatically by humans. Infect Immun 71, 1217-1224.

Peek, A. S., Souza, V., Eguiarte, L. E. \& Gaut, B. S. (2001). The interaction of protein structure, selection, and recombination on the evolution of the type-1 fimbrial major subunit (fimA) from Escherichia coli. J Mol Evol 52, 193-204.

Polley, S. D. \& Conway, D. J. (2001). Strong diversifying selection on domains of the Plasmodium falciparum apical membrane antigen 1 gene. Genetics 158, 1505-1512.

Posada, D. \& Crandall, K. A. (1998). MODELTEST: testing the model of DNA substitution. Bioinformatics 14, 817-818.

Roberts, A., Nightingale, K., Jeffers, G., Fortes, E., Kongo, J. M. \& Wiedmann, M. (2006). Genetic and phenotypic characterization of Listeria monocytogenes lineage III. Microbiology 152, 685-693.

Rousseaux, S., Olier, M., Lemaitre, J. P., Piveteau, P. \& Guzzo, J. (2004). Use of PCR-restriction fragment length polymorphism of inlA for rapid screening of Listeria monocytogenes strains deficient in the ability to invade Caco-2 cells. Appl Environ Microbiol 70, 2180-2185.

Rozas, J. \& Rozas, R. (1999). DnaSP version 3: an integrated program for molecular population genetics and molecular evolution analysis. Bioinformatics 15, 174-175.

Sauders, B. D., Durak, M. Z., Fortes, E., Windham, K., Schukken, Y., Lembo, A. J., Jr, Akey, B., Nightingale, K. K. \& Wiedmann, M. (2006). Molecular characterization of Listeria monocytogenes from natural and urban environments. J Food Prot 69, 93-105.

Schubert, W. D., Urbanke, C., Ziehm, T., Beier, V., Machner, M. P., Domann, E., Wehland, J., Chakraborty, T. \& Heinz, D. W. (2002). Structure of internalin, a major invasion protein of Listeria monocytogenes, in complex with its human receptor E-cadherin. Cell 111, 825-836.

Segura, A., Hurtado, A., Duque, E. \& Ramos, J. L. (2004). Transcriptional phase variation at the $f l h B$ gene of Pseudomonas putida DOT-T1E is involved in response to environmental changes and suggests the participation of the flagellar export system in solvent tolerance. J Bacteriol 186, 1905-1909.

Snyder, A. \& Marquis, H. (2003). Restricted translocation across the cell wall regulates secretion of the broad-range phospholipase $\mathrm{C}$ of Listeria monocytogenes. J Bacteriol 185, 5953-5958.

Theiss, P. \& Wise, K. S. (1997). Localized frameshift mutation generates selective, high-frequency phase variation of a surface lipoprotein encoded by a mycoplasma $\mathrm{ABC}$ transporter operon. J Bacteriol 179, 4013-4022.

Tsai, Y. H., Orsi, R. H., Nightingale, K. K. \& Wiedmann, M. (2006). Listeria monocytogenes internalins are highly diverse and evolved by recombination and positive selection. Infect Genet Evol 6, 378-389.

Urwin, R., Holmes, E. C., Fox, A. J., Derrick, J. P. \& Maiden, M. C. (2002). Phylogenetic evidence for frequent positive selection and recombination in the meningococcal surface antigen PorB. Mol Biol Evol 19, 1686-1694.

Vazquez-Boland, J. A., Kuhn, M., Berche, P., Chakraborty, T., Dominguez-Bernal, G., Goebel, W., Gonzalez-Zorn, B., Wehland, J. \& Kreft, J. (2001). Listeria pathogenesis and molecular virulence determinants. Clin Microbiol Rev 14, 584-640.

Ward, T. J., Gorski, L., Borucki, M. K., Mandrell, R. E., Hutchins, J. \& Pupedis, K. (2004). Intraspecific phylogeny and lineage group identification based on the prfA virulence gene cluster of Listeria monocytogenes. J Bacteriol 186, 4994-5002.

Wiedmann, M., Bruce, J. L., Keating, C., Johnson, A. E., McDonough, P. L. \& Batt, C. A. (1997). Ribotypes and virulence gene polymorphisms suggest three distinct Listeria monocytogenes lineages with differences in pathogenic potential. Infect Immun 65, 2707-2716.

Wilson, R. L., Elthon, J., Clegg, S. \& Jones, B. D. (2000). Salmonella enterica serovars Gallinarum and Pullorum expressing Salmonella enterica serovar Typhimurium type 1 fimbriae exhibit increased invasiveness for mammalian cells. Infect Immun 68, 4782-4785.

Yang, Z. (1997). PAML: a program package for phylogenetic analysis by maximum likelihood. Comput Appl Biosci 13, 555-556.

Yang, Z. \& Nielsen, R. (2002). Codon-substitution models for detecting molecular adaptation at individual sites along specific lineages. Mol Biol Evol 19, 908-917.

Yang, Z., Nielsen, R., Goldman, N. \& Pedersen, A. M. K. (2000). Codon-substitution models for heterogeneous selection pressure at amino acid sites. Genetics 155, 431-449.

Yang, Z., Wong, W. S. \& Nielsen, R. (2005). Bayes empirical Bayes inference of amino acid sites under positive selection. Mol Biol Evol 22, 1107-1118.

Zhang, J., Nielsen, R. \& Yang, Z. (2005). Evaluation of an improved branch-site likelihood method for detecting positive selection at the molecular level. Mol Biol Evol 22, 2472-2479.

Edited by: T. Msadek 